\title{
Influence of Biopreservatives and Vacuuming Packaging on Sensory Quality of Milk Solids based Khoa during Frozen Storage $\left(-18 \pm 2^{\circ} \mathrm{C}\right)$
}

\author{
Vaquil, P.K. Bhardwaj and Surender Kumar* \\ Department of Livestock Products Technology, College of Veterinary Sciences, Lala Lajpat Rai University of Veterinary E Animal \\ Sciences, Hisar, INDIA \\ *Corresponding author: S Kumar; E-mail: hoodas246@gmail.com
}

Received: 23 Oct., 2020

Revised: 10 Dec., 2020

Accepted: 13 De., 2020

\begin{abstract}
The study was carried out to find out the effectiveness of biopreservatives and vacuum packaging on the shelf-life of milk solids based khoa. Khoa samples were prepared individually by combinations of Skim milk powder (SMP) with ghee and whey protein concentrates and SMP with cream (50\% fat). The control khoa sample was prepared utilizing full cream buffalo milk ( $6 \%$ fat). Natamycin at $10 \mathrm{ppm}$ and nisin at $100 \mathrm{IU} / \mathrm{g}$ were added when khoa was still hot $\left(85-90{ }^{\circ} \mathrm{C}\right)$. The samples without biopreservatives were packaged in simple and vacuum packaging. It was found that vacuum packaged as well as biopreservatives incorporated samples were organoleptically acceptable up to 5 months of storage at freezing temperature $\left(-18 \pm 2{ }^{\circ} \mathrm{C}\right)$.
\end{abstract}

\section{HIGHLIGHTS}

O Khoa is very parishable item.

(- Incorporation of biopreservatives as well as vacuum packaging improved the storage life of khoa.

Keywords: Traditional dairy products, Khoa, milk solids, bioreservatives, natamycin, nisin, freezing, storage

The regional and seasonal imbalances in milk production in India call for developing suitable technology for efficient use of surplus milk solids. Conversion of liquid milk into traditional dairy products increases the longevity of milk solids. Traditional milk products which developed from milk in order to preserve the milk solids in a concentrated form for extended period play a significant role in the economic, social, religious and nutritional values of the Indian masses from the time immemorial. Khoa is a heat desiccated traditional Indian dairy product prepared by concentration of milk in an open pan with continuous stirring and scrapping (Aneja et al., 2002). Khoa is a major intermediate base for a variety of sweets like burf, peda, kalakand, gulabjamun etc. (Soumya et al., 2015).

Food spoilage refers to the damage of the original nutritional value, texture and flavor of the food that eventually renders food harmful to consumer and unsuitable to eat. Milk based sweets during storage undergo several physical, biochemical and microbiological changes causing them unfit for human consumption (Londhe et al., 2012). Microbes may gain entry into food at any stage of processing right from the farm to till the food is reached to the final consumer like at the time of packaging, transport and storage etc, so it becomes imperative not only to take all kinds of preventive measures but also to evaluate at every stage (Agarwal and Rachappa, 2006).

The deteriorating agents of the food quality are oxygen contained in the air and microorganisms such as bacteria and moulds. Oxygen promotes several types of deteriorative reactions in foods including oxidation of fat and pigments browning and is the key element needed

How to cite this article: Vaquil, Bhardwaj, P.K. and Kumar, S. (2020). Influence of biopreservatives and vacuuming packaging on sensory quality of milk solids based khoa during frozen storage $\left(-18 \pm 2{ }^{\circ} \mathrm{C}\right) . J$. Anim. Res., 10(6): 1001-1008.

Source of Support: None; Conflict of Interest: None क्ष 
for the growth of common spoilage bacteria and fungi. Therefore, to increase the shelf life of foods, the package atmosphere should contain low concentration of residual oxygen (Sandhya, 2010).

The storage life of khoa can be enhanced by using different types of food preservatives and antimicrobial agents (Wadhawa et al., 1993). In order to achieve improved food safety against pathogens, food industry makes use of chemical preservatives. But, these chemicals had many drawbacks such as their proven toxicity, alteration of the organoleptic and nutritional properties of food. Moreover, recent consumer demands for safe and minimally processed products without additives. Thus, to harmonize consumer demands with the necessary safety standards, traditional means of controlling microbial spoilage and safety hazards in foods are being replaced by combinations of innovative technologies that include biological antimicrobial systems such as lactic acid bacteria (LAB) and/or their metabolites (Nath et al., 2013). In addition, the absence of proper packaging, the rate of chemical deterioration of khoa such as oxidation and browning increase. But, use of antioxidant, antimicrobial agent and packaging increase the shelf- life of Khoa.

The concentration of natamycin for yoghurt preservation had been suggested to be in the range of 5-10 ppm (Thomas and Delves, 2001). The use of natamycin as a natural preservative in dairy products and other foods had been approved in over sixty countries (Delves et al., 2005). The use of nisin as biopreservative had been widely investigated in a large variety of fresh and processed foods (Jung et al., 1992). Nisin is effective against several pathogenic Gram-positive bacteria such as Listeria monocytogenes and Clostridium botulinum, but also against some Gram negative pathogens such as Escherichia coli and Salmonella spp. Consequently, it was admitted into the European food additive list, where it was assigned the number E234 (EEC, 1983).

\section{MATERIALS AND METHODS}

\section{Place of study}

The present study was carried out in the Department of Livestock Products Technology, College of Veterinary Sciences, LUVAS, Hisar to develop khoa by utilizing milk solids and subsequent impact of vacuum packaging and biopreservatives to enhance the shelf life in frozen conditions $\left(-18 \pm 2{ }^{\circ} \mathrm{C}\right)$.

\section{Procurement of raw materials}

Ghee, cream ( $50 \%$ fat) and full cream buffalo milk ( $6 \%$ fat) were procured from experimental dairy plant, Department of LPT, LUVAS, Hisar. Skim milk powder (SMP), whey protein concentrates and low density polyethylene (LDPE) pouches were procured from local market, Hisar.

\section{Biopreservative}

Nisin and Natamycin were procured from reputed firms.

\section{Preparation of khoa using milk solids}

Khoa was standardized for fat by Pearson's square method as described by De (2004) to meet legal standard of fat (20\% fat on fresh basis and $30 \%$ fat on dry basis). Khoa samples were prepared separately by combinations of SMP with ghee and whey protein concentrates and SMP with cream (50\% fat).

For ghee based khoa; $680 \mathrm{~g}$ SMP (13.6\%), $300 \mathrm{~g}$ ghee $(6 \%)$ and $20 \mathrm{~g}(0.4 \%)$ pre-standardized whey protein concentrates were mixed and hot water $(80 \%)$ was added to make total weight $5 \mathrm{~kg}$. Similarly, for cream based khoa; 500 g SMP (10\%) + 480 g cream $(9.6 \%)$ were mixed and hot water $(80.4 \%)$ added to make total weight $5 \mathrm{~kg}$. Proper mixing and filtration was done followed by preheating up to $65{ }^{\circ} \mathrm{C}$. Then homogenization (double stage) and desiccation was carried out in gas operated semiautomatic machine. The control khoa sample was prepared utilizing full cream buffalo milk (6\% fat).

\section{Packaging}

Biopreservatives added khoa samples were simply packaged in low density polyethylene bag and sealed with sealing machine. But samples without bio preservative were packaged in two different packaging techniques i.e. simple packaging and vacuum packaging.

\section{Addition of biopreservatives}

Natamycin and nisin were used to increase the storage life of developed products. Natamycin at 10 ppm (Rabee and 
Heba, 2017) and nisin at $100 \mathrm{IU} / \mathrm{g}$ (Gupta et al., 1989) were added while khoa was still hot (about 85-90 ${ }^{\circ} \mathrm{C}$ ). Proper mixing was done in machine.

\section{Sensory evaluation during storage}

Sensory analysis of developed khoa samples was performed by using the nine point hedonic scale (Nelson and Trout, 1964). A semi trained panel evaluated the sensory attributes viz. colour and appearance, flavor, and overall acceptability at regular interval of 1 month at freezing temperature $\left(-18 \pm 2{ }^{\circ} \mathrm{C}\right)$. Hedonic scale as shown in table 1 was used:

Table 1: Scores under 9-point hedonic scale

\begin{tabular}{ll}
\hline Data under 9-point hedonic scale & Score \\
\hline Liked extremely & 9 \\
Liked very much & 8 \\
Liked moderately & 7 \\
Liked slightly & 6 \\
Neither liked nor disliked & 5 \\
Disliked slightly & 4 \\
Disliked moderately & 3 \\
Disliked very much & 2 \\
Disliked extremely & 1 \\
\hline
\end{tabular}

\section{STATISTICAL ANALYSIS}

Data were analyzed statistically on 'SPSS-16.0' (SPSS Inc., Chicago, II USA) software package as per standard methods (Snedecor and Cochran, 1994). The statistical significance was estimated at $5 \%$ level and evaluated with Duncan's Multiple Range Test.

\section{RESULT AND DISCUSSION}

\section{Colour and appearance scores}

It is revealed from table 2 that the colour and appearance scores of khoa stored at frozen temperature range from 8.25 to 8.50 (i.e. all treatments liked very much) on fresh basis. However, scores were statistically similar. On every progressive month of storage there was continuous decline in the colour and appearance scores of all treatments. There was no significant difference in colour and appearance score of all treatments on particular day till end of storage during frozen storage. Colour and appearance scores of all samples ranged from 7.33 to 7.83 (i.e. likes moderately) on $2^{\text {nd }}$ month of storage. Highest value was reported in $\mathrm{C}_{3}$ (7.83) while $\mathrm{T}_{5}$ revealed lowest score (7.33).

Table 2: Colour and appearance score of khoa during frozen storage $\left(-18 \pm 2{ }^{\circ} \mathrm{C}\right)($ Mean \pm S.E., $n=12)$

\begin{tabular}{|c|c|c|c|c|c|c|}
\hline \multirow[t]{2}{*}{ Sample } & \multicolumn{6}{|c|}{ Month } \\
\hline & $0^{\text {th }}$ & $1^{\text {st }}$ & $2^{\text {nd }}$ & $3^{\text {rd }}$ & $4^{\text {th }}$ & $5^{\text {th }}$ \\
\hline$\overline{\mathrm{C}_{1}}$ & $8.42 \pm 0.15^{\mathrm{Aa}}$ & $8.00 \pm 0.25^{\mathrm{Aab}}$ & $7.67 \pm 0.19^{\mathrm{Abc}}$ & $7.25 \pm 0.18^{\mathrm{Ac}}$ & $6.67 \pm 0.14^{\text {Acd }}$ & $6.41 \pm 0.19^{\mathrm{Ad}}$ \\
\hline $\mathrm{C}_{2}$ & $8.50 \pm 0.23^{\mathrm{Aa}}$ & $8.08 \pm 0.19^{\mathrm{Aab}}$ & $7.75 \pm 0.25^{\mathrm{Abc}}$ & $7.33 \pm 0.14^{\mathrm{Acd}}$ & $6.75 \pm 0.22^{\text {Ade }}$ & $6.50 \pm 0.20^{\mathrm{Ae}}$ \\
\hline $\mathrm{C}_{3}$ & $8.41 \pm 0.19^{\text {Аа }}$ & $8.17 \pm 0.21^{\mathrm{Aa}}$ & $7.83 \pm 0.24^{\mathrm{Aab}}$ & $7.50 \pm 0.20^{\mathrm{Ab}}$ & $6.92 \pm 0.15^{\mathrm{Ac}}$ & $6.58 \pm 0.23^{\mathrm{Ac}}$ \\
\hline $\mathrm{C}_{4}$ & $8.42 \pm 0.14^{\mathrm{Aa}}$ & $8.08 \pm 0.26^{\mathrm{Aab}}$ & $7.75 \pm 0.22^{\mathrm{Abc}}$ & $7.33 \pm 0.18^{\mathrm{Ac}}$ & $6.75 \pm 0.13^{\mathrm{Ad}}$ & $6.58 \pm 0.22^{\mathrm{Ad}}$ \\
\hline $\mathrm{T}_{1}^{4}$ & $8.33 \pm 0.18^{\mathrm{Aa}}$ & $7.92 \pm 0.19^{\text {Aab }}$ & $7.50 \pm 0.20^{\mathrm{Abc}}$ & $7.17 \pm 0.16^{\mathrm{Ac}}$ & $6.50 \pm 0.15^{\mathrm{Ad}}$ & $6.25 \pm 0.21^{\mathrm{Ad}}$ \\
\hline $\mathrm{T}_{2}$ & $8.42 \pm 0.15^{\mathrm{Aa}}$ & $8.00 \pm 0.17^{\text {Aab }}$ & $7.58 \pm 0.23^{\mathrm{Abc}}$ & $7.25 \pm 0.13^{\mathrm{Ac}}$ & $6.58 \pm 0.19^{\mathrm{Ad}}$ & $6.33 \pm 0.23^{\mathrm{Ad}}$ \\
\hline $\mathrm{T}_{3}$ & $8.33 \pm 0.14^{\mathrm{Aa}}$ & $8.08 \pm 0.23^{\mathrm{Aab}}$ & $7.67 \pm 0.22^{\mathrm{Abc}}$ & $7.42 \pm 0.22^{\mathrm{Ac}}$ & $6.75 \pm 0.21^{\mathrm{Ad}}$ & $6.42 \pm 0.22^{\mathrm{Ad}}$ \\
\hline $\mathrm{T}_{4}$ & $8.41 \pm 0.15^{\mathrm{Aa}}$ & $8.00 \pm 0.22^{\mathrm{Aab}}$ & $7.75 \pm 0.21^{\mathrm{Ab}}$ & $7.17 \pm 0.21^{\mathrm{Ac}}$ & $6.58 \pm 0.19^{\mathrm{Ad}}$ & $6.42 \pm 0.23^{\mathrm{Ad}}$ \\
\hline $\mathrm{T}_{5}$ & $8.25 \pm 0.17^{\mathrm{Aa}}$ & $7.83 \pm 0.20^{\mathrm{Aab}}$ & $7.33 \pm 0.19^{\mathrm{Abc}}$ & $7.08 \pm 0.19^{\mathrm{Ac}}$ & $6.17 \pm 0.20^{\mathrm{Ad}}$ & $6.00 \pm 0.25^{\mathrm{Ad}}$ \\
\hline $\mathrm{T}_{6}$ & $8.33 \pm 0.14^{\mathrm{Aa}}$ & $7.92 \pm 0.19^{\text {Aab }}$ & $7.42 \pm 0.19^{\mathrm{Abc}}$ & $7.17 \pm 0.17^{\mathrm{Ac}}$ & $6.25 \pm 0.17^{\mathrm{Ad}}$ & $6.08 \pm 0.29^{\mathrm{Ad}}$ \\
\hline $\mathrm{T}_{7}$ & $8.25 \pm 0.18^{\mathrm{Aa}}$ & $8.00 \pm 0.21^{\mathrm{Aab}}$ & $7.50 \pm 0.15^{\mathrm{Abc}}$ & $7.33 \pm 0.19^{\mathrm{Ac}}$ & $6.42 \pm 0.19^{\mathrm{Ad}}$ & $6.08 \pm 0.28^{\mathrm{Ad}}$ \\
\hline $\mathrm{T}_{8}$ & $8.33 \pm 0.22^{\mathrm{Aa}}$ & $7.92 \pm 0.19^{\mathrm{Aab}}$ & $7.41 \pm 0.14^{\mathrm{Abc}}$ & $7.17 \pm 0.21^{\mathrm{Ac}}$ & $6.33 \pm 0.14^{\mathrm{Ad}}$ & $6.17 \pm 0.29^{\mathrm{Ad}}$ \\
\hline
\end{tabular}

Mean with capital superscripts within column and small superscripts within the row differ significantly ( $\mathrm{P} \leq 0.05)$

$\mathrm{C}_{1}$ - simple aerobically packaged FCM (full cream milk) based khoa, $\mathrm{C}_{2}$ - vacuum packaged FCM (full cream milk) based khoa, $\mathrm{C}_{3}$ - natamycin treated FCM (full cream milk) based khoa, $\mathrm{C}_{4}$ - nisin treated FCM (full cream milk) based khoa, $\mathrm{T}_{1}$ - simple aerobically packaged smp and cream based khoa, $\mathrm{T}_{2}$ - vacuum packaged smp and cream based khoa, $\mathrm{T}_{3}$ - natamycin treated SMP (skimmed milk powder) and cream based khoa, $\mathrm{T}_{4}$ - nisin treated SMP (skimmed milk powder) and cream based khoa, $\mathrm{T}_{5}$ - simple aerobically packaged SMP (skimmed milk powder) and ghee based khoa, $\mathrm{T}_{6}$ - vacuum packaged SMP (skimmed milk powder) and ghee based khoa, $\mathrm{T}_{7}$ - natamycin treated SMP (skimmed milk powder) and ghee based khoa, $\mathrm{T}_{8}$ - nisin treated SMP (skimmed milk powder) and ghee based khoa 
On $3^{\text {rd }}$ month of storage sensory panelist awarded the highest colour and appearance score to $\mathrm{C}_{3}(7.50)$ while lowest to $\mathrm{T}_{5}$ (7.08), although all values were found statistically similar with respect to each others. But when compared with previous month ( $2^{\text {nd }}$ month) then only score of $\mathrm{T}_{4}$ increased significantly. On $4^{\text {th }}$ month, colour and appearance scores ranged from 6.17 to 6.92 and highest score was observed in $\mathrm{C}_{3}$ (6.92), however all other sample were found non-significantly lower value than $\mathrm{C}_{3}$. All khoa samples had range of colour and appearance score from 6.00 to 6.58 on $5^{\text {th }}$ month of storage. $T_{5}$ had score 6.00 which was found non-significantly lower than all other samples. However the all treatments were liked slightly at $5^{\text {th }}$ month of storage at frozen temperature.

There was significant decrease in colour and appearance of khoa with progression in storage period. This might be due to microbial growth (yeast and moulds), known to be responsible for discolouration and lipolytic defects in khoa (Goyal and Srinivasan, 1984). Microbial contamination produces undesirable effects e.g. change in colour, odour, taste and texture of the products. Yadav and Beniwal (2009) and Beniwal et al. (2009) also reported decline in colour and appearance during storage of peda and khoa respectively at $-15 \pm 1^{\circ} \mathrm{C}$. All natamycin treated khoa samples had higher colour and appearance scores which might be owing to lower yeast and mould counts.

Khoa samples packaged under vacuum condition had better colour and appearance scores than simple packaged sample. This might be due to vacuum packaging which did not allow atmospheric factors to distort the colour and appearance of khoa. Kumar et al. (2010) also noticed higher colour and appearance score in vacuum packaged khoa than normal packaged khoa. Khoa samples prepared by using milk solids had slightly lower colour and appearance score as compare to buffalo milk khoa. Voghra and Rajoria (1983) also reported lower colour score for khoa prepared from milk powder than buffalo milk khoa.

\section{Body and texture score}

Body and texture scores of all treatments were reported statistically similar to each other on every particular month of storage on $2^{\text {nd }}$ month. But on $3^{\text {rd }}$ month body and texture score of $\mathrm{T}_{5}(7.00)$ was observed significantly lower than $\mathrm{C}_{3}$ (7.83). All stored sample had body and texture score more than 6 on $4^{\text {th }}$ month of frozen storage which indicated that all samples were liked slightly. Body and texture score of $\mathrm{C}_{4}$ (6.83) was reported significantly higher than $\mathrm{T}_{5}(6.08)$ and $\mathrm{T}_{6}(6.17)$.

There were significant decreases in body and texture scores of khoa in each treatment with advancement in storage period which might be due to increase in microbial counts during storage. Microbial contamination produces undesirable effects e.g. change in colour, odour, taste and texture of the product (Goyal and Srinivasan, 1984). However body and texture of all treatments did not differ significantly on particular month of storage. Yadav and Beniwal (2009) and Beniwal et al. (2009) also reported decrease in body and texture score of peda and khoa during storage at $-15 \pm 1^{\circ} \mathrm{C}$.

All natamycin treated samples had higher body and texture scores, which might be due to lower yeast and mould counts. Those khoa samples which were packaged under vaccum condition also had better body and texture score than normal packaged sample as vacuum packaging did not allow atmospheric factors to distort the body and texture of khoa. Kumar et al. (2010) also noticed higher body and texture score in vacuum packaged khoa than normal packaged khoa during storage. Khoa samples prepared by using milk solids had slightly lower body and texture scores as compared to buffalo milk khoa.

\section{Flavor scores}

It is revealed from table 4 , that flavor scores of khoa samples ranged from 8.42 to 8.17 . There was nonsignificant difference in flavor scores of all treatment on $0^{\text {th }}$ month of storage and similar trend were observed till the $3^{\text {rd }}$ month of storage at frozen temperature. On $1^{\text {st }}$ month of storage flavor scores ranged 7.75 to 8.08 and the highest value was reported to $C_{3}(8.08)$ while lowest to $\mathrm{T}_{5}(7.75)$ although all value differed non significantly. Sensory panelist awarded highest flavor score to $C_{3}(7.67)$ and scores ranged 7.25 to 7.67 on $2^{\text {nd }}$ month of storage. On $3^{\text {rd }}$ month of storage flavor scores of all treatments were found statistically similar and scores for every sample were observed more than 7 which indicated the moderately acceptance of all khoa samples on that day. Sensory panelist awarded highest score $7.42\left(\mathrm{C}_{3}\right)$ while lowest $7.00\left(\mathrm{~T}_{5}\right)$.

There was significant decrease in flavor scores of khoa in each treatment during storage time. This might be due 
Table 3: Body and texture score of khoa during frozen storage $\left(-18 \pm 2^{\circ} \mathrm{C}\right)($ Mean \pm S.E, $)(n=12)$

\begin{tabular}{|c|c|c|c|c|c|c|}
\hline \multirow{2}{*}{ Sample } & \multicolumn{6}{|c|}{ Month } \\
\hline & $\overline{0^{\text {th }}}$ & $1^{\text {st }}$ & $2^{\text {nd }}$ & $3^{\text {rd }}$ & $4^{\text {th }}$ & $5^{\text {th }}$ \\
\hline$\overline{\mathrm{C}_{1}}$ & $8.42 \pm 0.15^{\mathrm{Aa}}$ & $8.17 \pm 0.16^{\text {Aab }}$ & $8.00 \pm 0.25^{\text {Aab }}$ & $7.67 \pm 0.35^{\mathrm{ABb}}$ & $6.58 \pm 0.15^{\mathrm{ABc}}$ & $6.17 \pm 0.17^{\mathrm{Ac}}$ \\
\hline $\mathrm{C}_{2}$ & $8.50 \pm 0.23^{\mathrm{Aa}}$ & $8.25 \pm 0.18^{\text {Aab }}$ & $8.08 \pm 0.26^{\text {Aab }}$ & $7.75 \pm 0.25^{\mathrm{ABb}}$ & $6.67 \pm 0.22^{\mathrm{ABc}}$ & $6.25 \pm 0.18^{\mathrm{Ac}}$ \\
\hline $\mathrm{C}_{3}$ & $8.42 \pm 0.19^{\mathrm{Aa}}$ & $8.33 \pm 0.19^{\mathrm{Aa}}$ & $8.17 \pm 0.24^{\mathrm{Aa}}$ & $7.83 \pm 0.30^{\mathrm{Aab}}$ & $6.83 \pm 0.11^{\mathrm{Ac}}$ & $6.33 \pm 0.19^{\mathrm{Ac}}$ \\
\hline $\mathrm{C}_{4}$ & $8.50 \pm 0.15^{\mathrm{Aa}}$ & $8.25 \pm 0.25^{\mathrm{Aab}}$ & $8.08 \pm 0.22^{\text {Aab }}$ & $7.75 \pm 0.25^{\mathrm{ABb}}$ & $6.75 \pm 0.13^{\mathrm{ABc}}$ & $6.25 \pm 0.25^{\mathrm{Ac}}$ \\
\hline $\mathrm{T}_{1}$ & $8.41 \pm 0.14^{\mathrm{Aa}}$ & $8.08 \pm 0.19^{\text {Aab }}$ & $7.83 \pm 0.30^{\text {Aab }}$ & $7.50 \pm 0.23^{\mathrm{ABb}}$ & $6.25 \pm 0.18^{\mathrm{ABc}}$ & $5.92 \pm 0.23^{\mathrm{ABCc}}$ \\
\hline $\mathrm{T}_{2}$ & $8.33 \pm 0.19^{\mathrm{Aa}}$ & $8.17 \pm 0.17^{\text {Aab }}$ & $7.92 \pm 0.29^{\mathrm{Aab}}$ & $7.58 \pm 0.19^{\mathrm{ABc}}$ & $6.50 \pm 0.20^{\mathrm{ABd}}$ & $6.00 \pm 0.21^{\mathrm{ABd}}$ \\
\hline $\mathrm{T}_{3}$ & $8.42 \pm 0.14^{\mathrm{Aa}}$ & $8.25 \pm 0.22^{\mathrm{Aab}}$ & $8.00 \pm 0.25^{\text {Aab }}$ & $7.75 \pm 0.25^{\mathrm{ABb}}$ & $6.75 \pm 0.17^{\mathrm{ABc}}$ & $6.08 \pm 0.23^{\mathrm{Ad}}$ \\
\hline $\mathrm{T}_{4}$ & $8.42 \pm 0.15^{\mathrm{Aa}}$ & $8.17 \pm 0.21^{\mathrm{Aab}}$ & $7.92 \pm 0.26^{\mathrm{Aab}}$ & $7.67 \pm 0.19^{\mathrm{ABb}}$ & $6.58 \pm 0.19^{\mathrm{ABc}}$ & $6.00 \pm 0.21^{\mathrm{ABd}}$ \\
\hline $\mathrm{T}_{5}$ & $8.25 \pm 0.18^{\mathrm{Aa}}$ & $7.92 \pm 0.19^{\mathrm{Aab}}$ & $7.58 \pm 0.25^{\mathrm{Abc}}$ & $7.00 \pm 0.17^{\mathrm{Bc}}$ & $6.08 \pm 0.23^{\mathrm{Bd}}$ & $5.17 \pm 0.27^{\mathrm{De}}$ \\
\hline $\mathrm{T}_{6}$ & $8.33 \pm 0.14^{\mathrm{Aa}}$ & $8.00 \pm 0.21^{\mathrm{Aab}}$ & $7.67 \pm 0.22^{\mathrm{Ab}}$ & $7.08 \pm 0.15^{\mathrm{Bc}}$ & $6.17 \pm 0.20^{\mathrm{Bd}}$ & $5.25 \pm 0.25^{\mathrm{CDe}}$ \\
\hline $\mathrm{T}_{7}$ & $8.25 \pm 0.17^{\mathrm{Aa}}$ & $8.08 \pm 0.19^{\mathrm{Aa}}$ & $7.83 \pm 0.17^{\mathrm{Aa}}$ & $7.25 \pm 0.18^{\mathrm{ABb}}$ & $6.42 \pm 0.19^{\mathrm{ABc}}$ & $5.33 \pm 0.26^{\mathrm{BCDd}}$ \\
\hline $\mathrm{T}_{8}$ & $8.33 \pm 0.22^{\mathrm{Aa}}$ & $8.00 \pm 0.21^{\mathrm{Aa}}$ & $7.75 \pm 0.22^{\mathrm{Aa}}$ & $7.17 \pm 0.17^{\mathrm{ABb}}$ & $6.25 \pm 0.13^{\mathrm{ABc}}$ & $5.25 \pm 0.25^{\mathrm{CDd}}$ \\
\hline
\end{tabular}

Mean with capital superscripts within column and small superscripts within the row differ significantly $(\mathrm{P} \leq 0.05)$

$\mathrm{C}_{1}$ - simple aerobically packaged FCM (full cream milk) based khoa, $\mathrm{C}_{2}$ - vacuum packaged FCM (full cream milk) based khoa, $\mathrm{C}_{3}$ - natamycin treated FCM (full cream milk) based khoa, $\mathrm{C}_{4}$ - nisin treated FCM (full cream milk) based khoa, $\mathrm{T}_{1}$ - simple aerobically packaged smp and cream based khoa, $\mathrm{T}_{2}$ - vacuum packaged smp and cream based khoa, $\mathrm{T}_{3}$ - natamycin treated SMP (skimmed milk powder) and cream based khoa, $\mathrm{T}_{4}$ - nisin treated SMP (skimmed milk powder) and cream based khoa, $\mathrm{T}_{5}$ - simple aerobically packaged SMP (skimmed milk powder) and ghee based khoa, $\mathrm{T}_{6}$ - vacuum packaged SMP (skimmed milk powder) and ghee based khoa, $\mathrm{T}_{7}$ - natamycin treated SMP (skimmed milk powder) and ghee based khoa, $\mathrm{T}_{8}$ - nisin treated SMP (skimmed milk powder) and ghee based khoa

Table 4: Flavor scores of khoa during frozen storage $\left(-18 \pm 2^{\circ} \mathrm{C}\right)($ Mean \pm S.E., $n=12)$

\begin{tabular}{|c|c|c|c|c|c|c|}
\hline \multirow[t]{2}{*}{ Sample } & \multicolumn{6}{|c|}{ Month } \\
\hline & $0^{\text {th }}$ & $1^{\text {st }}$ & $2^{\text {nd }}$ & $3^{\text {rd }}$ & $4^{\text {th }}$ & $5^{\text {th }}$ \\
\hline$\overline{C_{1}}$ & $8.33 \pm 0.19^{\mathrm{Aa}}$ & $8.00 \pm 0.21^{\mathrm{Aab}}$ & $7.50 \pm 0.23^{\mathrm{Abc}}$ & $7.25 \pm 0.21^{\mathrm{Ac}}$ & $6.58 \pm 0.15^{\mathrm{ABCd}}$ & $6.25 \pm 0.22^{\mathrm{ABCd}}$ \\
\hline $\mathrm{C}_{2}$ & $8.42 \pm 0.23^{\mathrm{Aa}}$ & $8.08 \pm 0.19^{\mathrm{Aab}}$ & $7.58 \pm 0.22^{\mathrm{Abc}}$ & $7.33 \pm 0.14^{\mathrm{Ac}}$ & $6.67 \pm 0.23^{\mathrm{ABCd}}$ & $6.33 \pm 0.19^{\mathrm{ABCd}}$ \\
\hline $\mathrm{C}_{3}$ & $8.41 \pm 0.19^{\mathrm{Aa}}$ & $8.17 \pm 0.24^{\mathrm{Aa}}$ & $7.67 \pm 0.19^{\mathrm{Ab}}$ & $7.42 \pm 0.15^{\mathrm{Ab}}$ & $6.83 \pm 0.11^{\mathrm{Ac}}$ & $6.58 \pm 0.15^{\mathrm{Ac}}$ \\
\hline $\mathrm{C}_{4}$ & $8.42 \pm 0.15^{\mathrm{Aa}}$ & $8.08 \pm 0.23^{\mathrm{Aab}}$ & $7.58 \pm 0.19^{\mathrm{Abc}}$ & $7.33 \pm 0.19^{\mathrm{Ac}}$ & $6.75 \pm 0.13^{\mathrm{ABd}}$ & $6.50 \pm 0.20^{\mathrm{ABd}}$ \\
\hline $\mathrm{T}_{1}$ & $8.25 \pm 0.18^{\mathrm{Aa}}$ & $7.92 \pm 0.19^{\mathrm{Aa}}$ & $7.33 \pm 0.18^{\mathrm{Ab}}$ & $7.08 \pm 0.15^{\mathrm{Ab}}$ & $6.33 \pm 0.14^{\mathrm{ABCc}}$ & $6.00 \pm 0.17^{\mathrm{ABCc}}$ \\
\hline $\mathrm{T}_{2}$ & $8.42 \pm 0.15^{\mathrm{Aa}}$ & $8.00 \pm 0.17^{\mathrm{Aa}}$ & $7.42 \pm 0.23^{\mathrm{Ab}}$ & $7.17 \pm 0.11^{\mathrm{Ab}}$ & $6.50 \pm 0.20^{\mathrm{ABCc}}$ & $6.08 \pm 0.19^{\mathrm{ABCc}}$ \\
\hline $\mathrm{T}_{3}$ & $8.33 \pm 0.14^{\mathrm{Aa}}$ & $8.08 \pm 0.23^{\mathrm{Aab}}$ & $7.58 \pm 0.20^{\mathrm{Abc}}$ & $7.25 \pm 0.18^{\mathrm{Ac}}$ & $6.67 \pm 0.19^{\mathrm{ABCd}}$ & $6.33 \pm 0.14^{\mathrm{ABCd}}$ \\
\hline $\mathrm{T}_{4}$ & $8.42 \pm 0.15^{\mathrm{Aa}}$ & $8.00 \pm 0.25^{\mathrm{Aab}}$ & $7.50 \pm 0.15^{\mathrm{Abc}}$ & $7.17 \pm 0.21^{\mathrm{Ac}}$ & $6.58 \pm 0.19^{\mathrm{ABCd}}$ & $6.17 \pm 0.17^{\mathrm{ABCd}}$ \\
\hline $\mathrm{T}_{5}$ & $8.17 \pm 0.24^{\mathrm{Aa}}$ & $7.75 \pm 0.22^{\mathrm{Aab}}$ & $7.25 \pm 0.18^{\mathrm{Abc}}$ & $7.00 \pm 0.17^{\mathrm{Ac}}$ & $6.08 \pm 0.20^{\mathrm{Cd}}$ & $5.83 \pm 0.21^{\mathrm{Cd}}$ \\
\hline $\mathrm{T}_{6}$ & $8.33 \pm 0.14^{\mathrm{Aa}}$ & $7.83 \pm 0.17^{\mathrm{Ab}}$ & $7.33 \pm 0.19^{\mathrm{Ac}}$ & $7.08 \pm 0.15^{\mathrm{Ac}}$ & $6.17 \pm 0.17^{\mathrm{BCd}}$ & $5.92 \pm 0.19^{\mathrm{BCd}}$ \\
\hline $\mathrm{T}_{7}$ & $8.25 \pm 0.18^{\mathrm{Aa}}$ & $7.92 \pm 0.19^{\mathrm{Aab}}$ & $7.42 \pm 0.15^{\mathrm{Abc}}$ & $7.17 \pm 0.17^{\mathrm{Ac}}$ & $6.33 \pm 0.19^{\mathrm{ABCd}}$ & $6.08 \pm 0.19^{\mathrm{ABCd}}$ \\
\hline $\mathrm{T}_{8}$ & $8.33 \pm 0.23^{\mathrm{Aa}}$ & $7.83 \pm 0.20^{\mathrm{Aab}}$ & $7.33 \pm 0.18^{\mathrm{Abc}}$ & $7.00 \pm 0.17^{\mathrm{Ac}}$ & $6.25 \pm 0.13^{\mathrm{ABCd}}$ & $6.00 \pm 0.21^{\mathrm{ABCd}}$ \\
\hline
\end{tabular}

Mean with capital superscripts within column and small superscripts within the row differ significantly $(\mathrm{P} \leq 0.05)$

$\mathrm{C}_{1}$ - simple aerobically packaged FCM (full cream milk) based khoa, $\mathrm{C}_{2}$ - vacuum packaged FCM (full cream milk) based khoa, $\mathrm{C}_{3}$ - natamycin treated FCM (full cream milk) based khoa, $\mathrm{C}_{4}$ - nisin treated FCM (full cream milk) based khoa, $\mathrm{T}_{1}$ - simple aerobically packaged smp and cream based khoa, $T_{2}$ - vacuum packaged smp and cream based khoa, $T_{3}$ - natamycin treated SMP (skimmed milk powder) and cream based khoa, $\mathrm{T}_{4}$ - nisin treated SMP (skimmed milk powder) and cream based khoa, $\mathrm{T}_{5}$ - simple aerobically packaged SMP (skimmed milk powder) and ghee based khoa, $\mathrm{T}_{6}$ - vacuum packaged SMP (skimmed milk powder) and ghee based khoa, $\mathrm{T}_{7}$ - natamycin treated SMP (skimmed milk powder) and ghee based khoa, $\mathrm{T}_{8}$ - nisin treated SMP (skimmed milk powder) and ghee based khoa 
Table 5: Overall acceptability (OAA) scores of khoa during frozen storage $\left(-18 \pm 2{ }^{\circ} \mathrm{C}\right)(\mathrm{Mean} \pm \mathrm{S} . \mathrm{E}$., $\mathrm{n}=12)$

\begin{tabular}{|c|c|c|c|c|c|c|}
\hline \multirow{2}{*}{ Sample } & \multicolumn{6}{|c|}{ Month } \\
\hline & $0^{\text {th }}$ & $1^{\text {st }}$ & $2^{\text {nd }}$ & $3^{\text {rd }}$ & $4^{\text {th }}$ & $5^{\text {th }}$ \\
\hline $\mathrm{C}_{1}$ & $8.42 \pm 0.15^{\mathrm{Aa}}$ & $8.00 \pm 0.21^{\text {Aab }}$ & $7.75 \pm 0.25^{\mathrm{Ab}}$ & $7.42 \pm 0.19^{\mathrm{Ab}}$ & $6.58 \pm 0.14^{\mathrm{ABCc}}$ & $6.17 \pm 0.24^{\mathrm{ABc}}$ \\
\hline $\mathrm{C}_{2}$ & $8.50 \pm 0.23^{\mathrm{Aa}}$ & $8.08 \pm 0.19^{\mathrm{Aab}}$ & $7.83 \pm 0.23^{\mathrm{Abc}}$ & $7.50 \pm 0.15^{\mathrm{Ac}}$ & $6.92 \pm 0.22^{\mathrm{ABd}}$ & $6.25 \pm 0.25^{\mathrm{ABe}}$ \\
\hline $\mathrm{C}_{3}$ & $8.42 \pm 0.19^{\mathrm{Aa}}$ & $8.17 \pm 0.24^{\mathrm{Aab}}$ & $7.92 \pm 0.19^{\mathrm{Ab}}$ & $7.75 \pm 0.21^{\mathrm{Abc}}$ & $7.08 \pm 0.14^{\mathrm{Ac}}$ & $6.33 \pm 0.22^{\mathrm{Ad}}$ \\
\hline $\mathrm{C}_{4}$ & $8.42 \pm 0.15^{\mathrm{Aa}}$ & $8.08 \pm 0.23^{\mathrm{Aab}}$ & $7.83 \pm 0.20^{\mathrm{Ab}}$ & $7.58 \pm 0.23^{\mathrm{Abc}}$ & $6.92 \pm 0.19^{\mathrm{ABc}}$ & $6.25 \pm 0.25^{\mathrm{ABd}}$ \\
\hline $\mathrm{T}_{1}$ & $8.33 \pm 0.19^{\mathrm{Aa}}$ & $7.92 \pm 0.19^{\mathrm{Aa}}$ & $7.33 \pm 0.18^{\mathrm{Ab}}$ & $7.25 \pm 0.13^{\mathrm{Ab}}$ & $6.42 \pm 0.14^{\mathrm{ABCc}}$ & $6.08 \pm 0.26^{\mathrm{ABc}}$ \\
\hline $\mathrm{T}_{2}$ & $8.33 \pm 0.14^{\mathrm{Aa}}$ & $8.00 \pm 0.17^{\mathrm{Aa}}$ & $7.42 \pm 0.23^{\mathrm{Ab}}$ & $7.33 \pm 0.19^{\mathrm{Ab}}$ & $6.58 \pm 0.19^{\mathrm{ABCc}}$ & $6.17 \pm 0.20^{\mathrm{ABc}}$ \\
\hline $\mathrm{T}_{3}$ & $8.25 \pm 0.18^{\mathrm{Aa}}$ & $8.08 \pm 0.22^{\mathrm{Aab}}$ & $7.67 \pm 0.19^{\mathrm{Aab}}$ & $7.50 \pm 0.18^{\mathrm{Ab}}$ & $6.92 \pm 0.22^{\mathrm{ABc}}$ & $6.25 \pm 0.17^{\mathrm{ABd}}$ \\
\hline $\mathrm{T}_{4}$ & $8.33 \pm 0.19^{\mathrm{Aa}}$ & $8.00 \pm 0.21^{\mathrm{Aab}}$ & $7.67 \pm 0.18^{\mathrm{Ab}}$ & $7.42 \pm 0.19^{\mathrm{Ab}}$ & $6.67 \pm 0.18^{\mathrm{ABc}}$ & $6.17 \pm 0.20^{\mathrm{ABc}}$ \\
\hline $\mathrm{T}_{5}$ & $8.25 \pm 0.18^{\mathrm{Aa}}$ & $7.75 \pm 0.18^{\mathrm{Aab}}$ & $7.25 \pm 0.17^{\mathrm{Abc}}$ & $7.08 \pm 0.20^{\mathrm{Ac}}$ & $6.17 \pm 0.20^{\mathrm{Cd}}$ & $5.92 \pm 0.19^{\mathrm{Bd}}$ \\
\hline $\mathrm{T}_{6}$ & $8.25 \pm 0.19^{\mathrm{Aa}}$ & $7.83 \pm 0.17^{\mathrm{Aab}}$ & $7.33 \pm 0.19^{\mathrm{Abc}}$ & $7.17 \pm 0.17^{\mathrm{Ac}}$ & $6.25 \pm 0.17^{\mathrm{Cd}}$ & $6.00 \pm 0.21^{\mathrm{Bd}}$ \\
\hline $\mathrm{T}_{7}$ & $8.17 \pm 0.17^{\mathrm{Aa}}$ & $7.92 \pm 0.23^{\mathrm{Aab}}$ & $7.42 \pm 0.15^{\mathrm{Abc}}$ & $7.33 \pm 0.19^{\mathrm{Ac}}$ & $6.50 \pm 0.19^{\mathrm{ABCd}}$ & $6.17 \pm 0.20^{\mathrm{ABd}}$ \\
\hline $\mathrm{T}_{8}$ & $8.25 \pm 0.22^{\mathrm{Aa}}$ & $7.83 \pm 0.17^{\mathrm{Aab}}$ & $7.42 \pm 0.14^{\mathrm{Abc}}$ & $7.25 \pm 0.18^{\mathrm{Ac}}$ & $6.33 \pm 0.14^{\mathrm{BCd}}$ & $6.00 \pm 0.21^{\mathrm{Bd}}$ \\
\hline
\end{tabular}

Mean with capital superscripts within column and small superscripts within the row differ significantly $(\mathrm{P} \leq 0.05)$

$\mathrm{C}_{1}$ - simple aerobically packaged FCM (full cream milk) based khoa, $\mathrm{C}_{2}$ - vacuum packaged FCM (full cream milk) based khoa, $\mathrm{C}_{3}$ - natamycin treated FCM (full cream milk) based khoa, $\mathrm{C}_{4}$ - nisin treated FCM (full cream milk) based khoa, $\mathrm{T}_{1}$ - simple aerobically packaged smp and cream based khoa, $\mathrm{T}_{2}$ - vacuum packaged smp and cream based khoa, $\mathrm{T}_{3}$ - natamycin treated SMP (skimmed milk powder) and cream based khoa, $\mathrm{T}_{4}$ - nisin treated SMP (skimmed milk powder) and cream based khoa, $\mathrm{T}_{5}-$ simple aerobically packaged SMP and ghee based khoa, $\mathrm{T}_{6}-$ vacuum packaged SMP (skimmed milk powder) and ghee based khoa, $\mathrm{T}_{7}$ - natamycin treated SMP (skimmed milk powder) and ghee based khoa, $\mathrm{T}_{8}$ - nisin treated SMP (skimmed milk powder) and ghee based khoa

to increase in microbial counts during storage. Microbial contamination produces undesirable effects e.g. change in colour, odour, taste and texture of the product (Goyal and Srinivasan, 1984). Our results were accordance with Yadav and Beniwal (2009) and Beniwal et al. (2009) also reported decrease in flavor during storage of peda and khoa at $-15 \pm 1^{\circ} \mathrm{C}$. All natamycin treated khoa samples had higher flavor scores; this might be due to lower yeast and mould counts.

Those khoa samples which were packaged under vacuum condition had better flavor score than normal packaged sample. Vacuum packaging did not allow atmospheric factors to distort the sensory attributes of khoa. Kumar et al. (2010) also noticed the higher colour and appearance score in vacuum packaged khoa than normal packaged khoa at refrigerated temperature. Khoa samples prepared by using milk solids had slightly lower flavor scores as compared to buffalo milk khoa. Voghra and Rajoria (1983) also reported sensory score for khoa prepared from milk powder than buffalo milk khoa.

\section{Overall acceptability scores (OAA)}

Sensory panelist awarded highest overall acceptability score to $C_{2}(8.50)$ while lowest to $T_{7}(8.17)$ on fresh basis. No significant difference was observed in OAA scores among all samples on 0 day and same trend was followed on $1^{\text {st }}$ and $2^{\text {nd }}$ month of storage. On $1^{\text {st }}$ month, the OAA scores ranged from 7.92 to 8.17. OAA score of $T_{5}$ was noticed lowest which differed non significantly among all treatments. OAA scores of all samples were found more than 7 (i.e. liked moderately) on $2^{\text {nd }}$ month of storage. Sensory panelist awarded the highest score to $C_{3}$ (7.92) and lowest to $\mathrm{T}_{5}(7.25)$.

On $3^{\text {rd }}$ month the OAA scores of khoa samples ranged from 7.08 to 7.75 and all samples had statistically similar values. Sensory panelist awarded OAA scores ranged 6.17 to 7.08 on $4^{\text {th }}$ month during frozen storage study. OAA score of $\mathrm{C}_{3}$ (7.08) was found significantly higher than $\mathrm{T}_{5}$ (6.17), $\mathrm{T}_{6}(6.25)$ and $\mathrm{T}_{8}(6.33)$. On $5^{\text {th }}$ month the OAA scores of all khoa samples were reported more than 6 except $T_{5}(5.92) . C_{3}(6.33)$ had significantly higher score 
than $\mathrm{T}_{5}(5.92)$, $\mathrm{T} 6$ (6.00) and $\mathrm{T}_{8}(6.00)$. All samples were slightly liked on $5^{\text {th }}$ month of storage except $T_{5}$ which was neither liked nor disliked.

There were significant decreases in OAA scores of khoa for each treatment during storage time. This might be due to microbial growth responsible for discolouration and lipolytic defects in khoa (Goyal and Srinivasan, 1984). Microbial contamination exerts undesirable effects e.g. change in colour, odour, taste and texture of the product. However OAA of all treatments did not differ significantly on each same day of storage. Yadav and Beniwal (2009) and Beniwal et al. (2009) also reported decrease in OAA score during storage of peda and khoa at $-15 \pm 1^{\circ} \mathrm{C}$.

Natamycin as well nycin treated samples had higher OAA scores; this might be due to lower yeast and mould counts. Those khoa samples which were packaged under vacuum condition had better OAA score than normal packaged sample. Kumar et al. (2010) and Sharma et al. (2001) also noticed higher OAA score in vacuum packaged khoa than normal packaged khoa during storage. Khoa samples prepared by using milk solids had slightly lower OAA scores as compared to buffalo milk khoa.

\section{CONCLUSION}

The present study revealed that the milk solids (SMP, cream and ghee) could be very well suited to develop khoa which was comparable to khoa prepared from full cream buffalo milk. Vacuum packaging, Natamycin at $10 \mathrm{ppm}$ and nisin at $100 \mathrm{IU} / \mathrm{g}$ were equally effective in maintaining the sensory quality of milk solids incorporated khoa up to 5 months in frozen storage.

\section{ACKNOWLEDGEMENTS}

The authors are thankful to Head of Department, Department of Livestock Products Technology, College of Veterinary Sciences, Lala Lajpat Rai University of Veterinary \& Animal Sciences, Hisar for providing necessary facilities.

\section{REFERENCES}

Agarwal R. and Rachappa S.B. 2006. Finished product quality and safety. All India Dairy Business Directory, pp. 97-105.
Aneja R.P., Mathur B.N., Chandan R.C. and Banerjee A.K. 2002. Technology of Indian Milk Products, Dairy India Publications Delhi, pp. 122-125.

Beniwal, B.S., Rai D.C., Kapoor, C.M. and Bhardwaj, P.K. 2009. Effects of packaging material on keeping quality of khoa produced under semi-automatic process. Pakistan $J$. Zool. Suppl., 9: 233-238.

Delves, B.J., Thomas, L.V., Doan, C.H. and Davidson, P.M 2005. Natamycin. In: Antimicrobials in Food, Third Edition. Davidson P.M., Sofos J.N., Branen A.L. (eds). CRC Press; Tailor and Francis Group, Baca-Raton-USA, pp. 275-287.

EEC. 1983. EEC Commission Directive. 83/463/EEC.

Goyal, G.K. and. Srinivasan, M.R 1984. Microbiological quality of fresh khoa samples. Asian J. Dairy Res., 3: 114-116.

Gupta, R.K., Prasad, N. and Prasad, D.N. 1989. Use of nisin in dairy industry. Indian Dairyman, 41(7): 229-233.

Jung, D.S., Bodyfelt, F.W. and Daeschel, M.A. 1992. Influence of fat and emulsifiers on the efficacy of nisin in inhibiting Listeria monocytogenes in fluid milk. J. Dairy Sci., 75: 387393.

Kumar, M., Beniwal, B.S. and Rai, D.C. 2010. Effects of antioxidants on shelf life of khoa under refrigerated conditions. Egyptian J. Dairy Sci., 38: 211-218.

Londhe, G.K., Pal, D. and Raju, P.N. 2012. Effect of packaging techniques on shelf life of brown peda, a milk based confection. J. Food Sci. Technol., 47: 117-125.

Nath, S., Chowdhury, S., Sarkar, S. and Dora, K.C. 2013. Lactic Acid Bacteria - A Potential biopreservative in sea food industry. Int. J. Adv. Res., 1(6): 471-475.

Nelson, J.A. and. Trout, G.M. 1964. Judging Dairy Porducts $4^{\text {th }}$ Edn. Olson Publ. Co. Nilwankee, pp. 296.

Rabee, A.O. and Heba, H.S. 2017. The inhibitory Effect of Natamycin and Potassium Sorbate on Mold Growth in Egyptian Fresh Soft Cheese (Tallaga Cheese). Alexandria J. Veterinary Sci., 53(2): 33-37.

Sandhya, 2010. Modified atmosphere packaging of fresh produce: current status and future needs. LWT Food Sci. Technol., 43: 381-392.

Sharma, H.K., Singhal, R.S. and Kulkarni, P.R. 2001. Effect of packaging under vacuum or under nitrogen on the keeping quality of danedar khoa. Int. J. Dairy Technol., 54(3): 107110.

Snedecor, G.W. and Cochran, W.G. 1994. Statistical method, $8^{\text {th }}$ edition, Oxford and IBAS publishing Company Calcutta, pp. 172-196.

Soumya, R., Indumathi K.P., Arora, S., Sharma, V. and Singh, A.K. 2015. Detection of calcium based neutralizers in milk 
and milk products by AAS. J. Food Sci. Technol., 52(2): 1188-1193.

Thomas, L.V. and Delves, B.J. 2001. Applications of the natural food preservative natamycin. Res. Advan. Food Sci., 2: 1-10.

Vogrha, V.R. and Rajorhia, G.S. 1983. Utilization of dried milk for khoa making. Asian J. Dairy Res., 2(2): 113-118.
Wadhwa, B.K., Gandhi D.N. and Goyal G.K. 1993. Enhancement in the shelf life of khoa. Indian Food Pecker, 47: 50-53.

Yadav, R. and Beniwal, B.S. 2009. Effect of antioxidants and preservative on keeping quality of peda stored at sub-zero temperature. J. Dairying Foods Home Sci., 28(4): 164-169. 